PAPERs in Physics, VOL. 8, ART. 080008 (2016)

www.papersinphysics.org

Received: 6 September 2016, Accepted: 5 November 2016

Edited by: J. P. Paz

Licence: Creative Commons Attribution 3.0

DOI: http://dx.doi.org/10.4279/PIP.080008

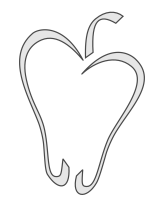

ISSN 1852-4249

\title{
Green's functions technique for calculating the emission spectrum in a quantum dot-cavity system
}

\begin{abstract}
Edgar A. Gómez, ${ }^{1 *}$ J. D. Hernández-Rivero, ${ }^{2}$ Herbert Vinck-Posada ${ }^{3}$
We introduce the Green's functions technique as an alternative theory to the quantum regression theorem formalism for calculating the two-time correlation functions in open quantum systems at the steady state. In order to investigate the potential of this theoretical approach, we consider a dissipative system composed of a single quantum dot inside a semiconductor cavity and the emission spectrum is computed due to the quantum dot as well as the cavity. We propose an algorithm based on the Green's functions technique for computing the emission spectrum that can easily be adapted to more complex open quantum systems. We found that the numerical results based on the Green's functions technique are in perfect agreement with the quantum regression theorem formalism. Moreover, it allows overcoming the inherent theoretical difficulties associated with the direct application of the quantum regression theorem in open quantum systems.
\end{abstract}

\section{Introduction}

The measurement and control of light produced by quantum systems have been the focus of interest of the cavity quantum electrodynamics $[1,2]$. Specially, the emission of light powered by solid-state devices coupled to nanocavities is an extensive area of research due to its promising technological applications, such as infrared and low-threshold lasers $[3,4]$, single and entangled photon sources $[5,6]$, as well as various applications in quantum cryptography [7] and quantum information theory [8]. Experiments with semiconductor quantum dots (QDs)

*E-mail: eagomez@uniquindio.edu.co

1 Universidad del Quindío, A.A. 2639, 630004 Armenia, Colombia.

2 Departamento de Física, Universidade Federal de Minas Gerais, A.A. 486, 31270-901 Pampulha, Belo Horizonte, Minas Gerais, Brazil.

3 Universidad Nacional de Colombia, A.A. 055051, 111321 Bogotá, Colombia. embedded in microcavities have revealed a plethora of quantum effects and offer desirable properties for harnessing coherent quantum phenomena at the single photon level. For example, the Purcell enhancement [9], photon antibunching [10], vacuum Rabi splitting [11] and strong light matter coupling [12]. These and many other quantum phenomena are being confirmed experimentally by observing the power spectral density of the light (PSD) emitted by the quantum-dot cavity systems (QDcavity). Thus, the PSD, or the so-called emission spectrum of the system, becomes the only relevant information that allows to study the properties of the light via measurements of correlation functions, as it is stated by the Wiener-Khintchine theorem [13]. In order to compute the emission spectrum of the QD-cavity systems in the framework of open quantum systems, different approaches have been elaborated from the theoretical point of view. For example, the method of thermodynamic Green's functions has been applied to the determination of the susceptibilities and absorption spectrum of 
Papers in Physics, vol. 8, ART. 080008 (2016) / E. A. Gómez et al.

atomic systems embedded in nanocavities [14], the time-resolved photo-luminescence approach whose application allows to determine the emission spectrum when an additional subsystem is considered, the so-called the photon reservoir [15]. These theoretical approaches are based on several approximations and therefore, they have their own limitations when they are considered in more general scenarios. In consequence, these methods are not used extensively.

Frequently, the emission spectrum of QD-cavity systems is computed through the Quantum Regression Theorem (QRT) [16-18], since it relates the evolution of mean values of observables and the two-time correlation functions. It is worth mentioning that the QRT approach can be difficult to implement in a computer program because computational complexity increases significantly as the number of QDs or modes inside the cavity are being considered; more precisely, the dimensionality associated with the Hilbert space is large. In general, the QRT approach is time-consuming because it is required to solve a large system of coupled differential equations and numerical instabilities that can arise. Moreover, theoretical complications related to dynamics of the operators involved can appear, as we will point out in the next section. In spite of this, the QRT approach is widely used in theoretical works, for example, in studies about photoluminescence spectra of coupled light-matter systems in microcavities in the presence of a continuous and incoherent pumping [19,20]. Also, in studies considering the relation between dynamical regimes and entanglement in QD-cavity systems [21,22]. In the past, the Green's functions technique (GFT) was successfully applied for calculating the emission spectrum for a very simple quantum system, e.g., the micro-maser [23]. Nevertheless, this approach has not been widely noticed in many significant situations in open quantum systems. The purpose of this work is to provide a simple and efficient numerical method based on the GFT in order to overcome the inherent difficulties associated with the direct application of the QRT approach by solving the dynamics of the system in the frequency domain directly. This paper is structured as follows: the theoretical background of the QRT as well as the GFT are presented in section II. A concrete application of our proposed methodology for calculating the emission spectrum of the QD- cavity system is considered in section III. Moreover, for comparison purposes with the GFT, we discuss in some detail the methodology of the QRT for calculating the emission spectrum of the cavity. The numerical results for the emission spectrum of the quantum dot, as well as of the cavity obtained from both the GFT and the QRT, are shown in section IV. A discussion about our findings is summarized in section $\mathrm{V}$.

\section{Theoretical background}

\section{i. Quantum regression theorem}

One of the most important measurements when the light excites resonantly a QD-cavity system is the emission spectrum of the system. From a theoretical point of view, it is assumed that it corresponds to a stationary and ergodic process which can be calculated as a PSD of light by using the well-known Wiener-Khintchine theorem [13]. This theorem states that the emission spectrum is given by the Fourier Transform of the two-time correlation function of the operator field $\hat{a}$; explicitly, it is

$$
S(\omega)=\mathfrak{R e} \lim _{t \rightarrow \infty} \int_{0}^{\infty}\left\langle\hat{a}^{\dagger}(t+\tau) \hat{a}(t)\right\rangle e^{i \omega \tau} d \tau .
$$

In order to calculate the two-time correlation function involved in Eq. (1), a theoretical approach based on the QRT is frequently considered. It states that if a set of operators $\left\{\hat{O}_{i}(t+\right.$ $\tau)\}$ satisfy the dynamical equations $\frac{d}{d \tau}\left\langle\hat{O}_{i}(t+\right.$ $\tau)\rangle=\sum_{j} L_{i j}\left\langle\hat{O}_{j}(t+\tau)\right\rangle$ then $\frac{d}{d \tau}\left\langle\hat{O}_{i}(t+\tau) \hat{O}(t)\right\rangle=$ $\sum_{j} L_{i j}\left\langle\hat{O}_{j}(t+\tau) \hat{O}(t)\right\rangle$ is valid for any operator $\hat{O}(t)$ at an arbitrary time $t$. Here, $L_{i j}$ represents the matrix of coefficients associated with the coupled linear equations of motion. It is worth mentioning that validity of this theorem holds whenever a closed set of operators is associated with the dynamics. In general, to obtain the closed set of operators can be difficult or an impossible task, since there must be added as many operators as necessary in order to close the dynamics of the system. For example, to calculate the emission spectrum in a model of QD-cavity system [20,21], two new operators are required because the field operators in the interaction picture do not lead to a complete set. 


\section{ii. Green's functions technique}

Let us consider a QD-cavity system and an operator $\hat{A}$ which does not operate on the reservoirs, then its single-time expectation value in the Heisenberg representation is given by

$$
\langle\hat{\tilde{A}}(t+\tau)\rangle=\operatorname{Tr}_{S \otimes R}\left[\hat{\tilde{A}}(t+\tau) \hat{\tilde{\rho}}_{S \otimes R}(t)\right] .
$$

The density operator system-reservoir can be evolved from an initial state at time 0 to an arbitrary time $t$ via $\hat{\tilde{\rho}}_{S \otimes R}(t)=\hat{U}^{\dagger}(t, 0) \hat{\rho}_{S \otimes R}(0) \hat{U}(t, 0)$, with $\hat{U}(t, 0)$ being a unitary time-evolution operator involving the Hamiltonian terms of the system and reservoirs. Moreover, the operator $\hat{\tilde{\rho}}_{S \otimes R}(t)=$ $\hat{\tilde{\rho}}_{S}(t) \otimes \hat{\tilde{\rho}}_{R}(t)$ depicts the composite density operator of the system and reservoir. It is worth pointing out that tilde means that the operator has been transformed to the Heisenberg representation and that the dynamics of the system depend directly on $\hat{\tilde{\rho}}_{S \otimes R}(t)$ for all times. The validity of the Markovian approximation requires that the state of the system is sufficiently well described when it is considered that $\hat{\tilde{\rho}}_{S}(t)=\operatorname{Tr}_{R}\left(\hat{\tilde{\rho}}_{S \otimes R}(t)\right)$. Therefore, it is sufficient to write $\hat{\tilde{\rho}}_{S \otimes R}(t)=\tilde{\tilde{\rho}}_{S}(t) \otimes \hat{\tilde{\rho}}_{R}(t)$ for all times. If we assume that at $t=0$ the initial state of the system is the steady state, then $\hat{\tilde{\rho}}_{S \otimes R}(0)=\hat{\rho}_{S \otimes R}^{(s)}$. Here, the superscript "(ss)" should be understood to be the steady state of the system-reservoir. After tracing over degrees of freedom of the reservoirs, we have that the Eq. (2) takes the form

$$
\langle\hat{\tilde{A}}(\tau)\rangle=\operatorname{Tr}_{S}\left[\hat{\tilde{A}}(0) \hat{\tilde{\rho}}_{S}(\tau)\right],
$$

where the reduced density operator for the system is given by $\hat{\tilde{\rho}}_{S}(\tau)=\operatorname{Tr}_{R}\left[\hat{U}(\tau, 0) \hat{\tilde{\rho}}_{S \otimes R}(0) \hat{U}^{\dagger}(\tau, 0)\right]$ and $\hat{\tilde{A}}(0)=\hat{A}$. If $\hat{\tilde{\rho}}_{S}(\tau)$ satisfies the Lindblad master equation $d \hat{\tilde{\rho}}_{S}(\tau) / d \tau=\mathcal{L} \hat{\tilde{\rho}}_{S}(\tau)$ with $\mathcal{L}$ the superoperator defined as $\mathcal{L} \hat{\tilde{\rho}}_{S}(\tau)=-i\left[\hat{H}_{S}, \hat{\tilde{\rho}}_{S}(\tau)\right]+$ $\sum_{j} \frac{\Gamma_{j}}{2}\left(2 \hat{X}_{j} \hat{\tilde{\rho}}_{S}(\tau) \hat{X}_{j}^{\dagger}-\hat{X}_{j}^{\dagger} \hat{X}_{j} \hat{\tilde{\rho}}_{S}(\tau)-\hat{\tilde{\rho}}_{S}(\tau) \hat{X}_{j}^{\dagger} \hat{X}_{j}\right)$ for an operator $\hat{X}_{j}$, then the expectation value $\langle\hat{\tilde{A}}(\tau)\rangle$ can be computed by solving the dynamics associated to the Lindblad master equation. It is worth mentioning that the Hamiltonian operator $\hat{H}_{S}$ describes the QD-cavity system and $\Gamma_{j}$ corresponds to the damping (pumping) rate associated to the operator $\hat{X}_{j}$.

In order to calculate the two-time correlation function $\langle\hat{\tilde{A}}(t+\tau) \hat{\tilde{B}}(t)\rangle$ where $\hat{\tilde{A}}(t+\tau)=\hat{U}^{\dagger}(t+$ $\tau, t) \hat{\tilde{A}}(t) \hat{U}(t+\tau, t)$ and $\hat{\tilde{B}}(t)=\hat{U}^{\dagger}(t, 0) \hat{B} \hat{U}(t, 0)$ are arbitrary Heisenberg operators which do not operate on the reservoirs, we proceed similarly to the case of the single-time expectation value, it is

$$
\begin{aligned}
\langle\hat{\tilde{A}}(\tau) \hat{\tilde{B}}(0)\rangle & =\operatorname{Tr}_{S \otimes R}\left[\hat{\tilde{A}}(\tau) \hat{\tilde{B}}(0) \hat{\tilde{\rho}}_{S \otimes R}(0)\right], \\
& =\operatorname{Tr}_{S}[\hat{\tilde{A}}(0) \hat{\tilde{G}}(\tau)],
\end{aligned}
$$

where we have used the well-known properties of the unitary time-evolution operator and the fact that the system at time $t=0$ is in the steady state. We have defined the operator

$$
\hat{\tilde{G}}(\tau)=\operatorname{Tr}_{R}\left[\hat{U}(\tau, 0) \hat{\tilde{B}}(0) \hat{\tilde{\rho}}_{S \otimes R}(0) \hat{U}^{\dagger}(\tau, 0)\right]
$$

where the trace operation is performed on the reservoirs only. By performing the time derivation of Eq. (4), we have that

$$
\frac{d}{d \tau}\langle\hat{\tilde{A}}(\tau) \hat{\tilde{B}}(0)\rangle=\operatorname{Tr}_{S}\left[\hat{\tilde{A}}(0) \frac{d \hat{\tilde{G}}(\tau)}{d \tau}\right] .
$$

where

$$
\begin{aligned}
\frac{d \hat{\tilde{G}}(\tau)}{d \tau} & =\frac{d}{d \tau} \operatorname{Tr}_{R}\left[\hat{U}(\tau, 0) \hat{\tilde{B}}(0) \hat{\tilde{\rho}}_{S \otimes R}(0) \hat{U}^{\dagger}(\tau, 0)\right], \\
& =\frac{d}{d \tau} \operatorname{Tr}_{R}\left[\hat{\tilde{B}}(\tau) \hat{\tilde{\rho}}_{S \otimes R}(\tau)\right], \\
& =\operatorname{Tr}_{R}\left[\hat{\tilde{B}}(\tau) \frac{d \hat{\tilde{\rho}}_{S \otimes R}(\tau)}{d \tau}+\hat{\tilde{\rho}}_{S \otimes R}(\tau) \frac{d \hat{\tilde{B}}(\tau)}{d \tau}\right], \\
& =\operatorname{Tr}_{R}\left[\hat{\tilde{B}}(\tau) \frac{d \hat{\tilde{\rho}}_{S \otimes R}(\tau)}{d \tau}\right], \\
& +\operatorname{Tr}_{R}\left[\frac{d \hat{\tilde{B}}(\tau)}{d \tau} \hat{\tilde{\rho}}_{S \otimes R}(\tau)\right] .
\end{aligned}
$$

Notice that the last term vanishes since $\operatorname{Tr}_{R}\left[\frac{d \hat{\tilde{B}}(\tau)}{d \tau} \hat{\tilde{\rho}}_{S \otimes R}(\tau)\right]=\frac{d}{d \tau} \operatorname{Tr}_{R}\left[\hat{\tilde{B}}(0) \hat{\tilde{\rho}}_{S \otimes R}(0)\right]$ is independent of time $\tau$. Thus, the Eq. (7) can be reduced to the form

$$
\begin{aligned}
\frac{d \hat{\tilde{G}}(\tau)}{d \tau} & =\operatorname{Tr}_{R}\left[\frac{d \hat{\tilde{\rho}}_{S \otimes R}(\tau)}{d \tau} \hat{\tilde{B}}(\tau)\right] \\
& =\operatorname{Tr}_{R}\left[\mathcal{L} \hat{\tilde{\rho}}_{S \otimes R}(\tau) \hat{\tilde{B}}(\tau)\right] \\
& =\mathcal{L} \operatorname{Tr}_{R}\left[\hat{\tilde{\rho}}_{S \otimes R}(\tau) \hat{\tilde{B}}(\tau)\right] \\
& =\mathcal{L} \operatorname{Tr}_{R}\left[\hat{U}(\tau, 0) \hat{\tilde{B}}(0) \hat{\tilde{\rho}}_{S \otimes R}(0) \hat{U}^{\dagger}(\tau, 0)\right] \\
& =\mathcal{L} \hat{\tilde{G}}(\tau)
\end{aligned}
$$


where we have taken into account that the superoperator $\mathcal{L}$ acts only on the system Hilbert space and not on the reservoir. It is straightforward to conclude that $\hat{\tilde{G}}(\tau)$ is an operator that obeys the same dynamical equations as $\hat{\tilde{\rho}}_{S}(\tau)$. More precisely, $d \hat{\tilde{G}}(\tau) / d \tau=\mathcal{L} \hat{\tilde{G}}(\tau)$ with the boundary condition $\hat{\tilde{G}}(0)=\hat{\tilde{B}}(0) \hat{\tilde{\rho}}_{S}(0)$ at the steady state of the system.

We also conclude that the two-time correlation function in the long-time limit can be written as

$$
\lim _{t \rightarrow \infty}\langle\hat{\tilde{A}}(t+\tau) \hat{\tilde{B}}(t)\rangle=\operatorname{Tr}_{S}[\hat{A} \hat{\tilde{G}}(\tau)],
$$

where $\hat{\tilde{G}}(\tau)=\operatorname{Tr}_{R}\left[\hat{U}(\tau) \hat{B} \hat{\rho}_{S \otimes R}^{(s s)} \hat{U}^{\dagger}(\tau)\right]$ is defined as the Green's functions operator and the operators $\hat{A}$, $\hat{B}$ and $\hat{\rho}_{S \otimes R}^{(s s)}$ are in the Schrödinger representation. $\hat{A}=\hat{\tilde{A}}(0)$ and $\hat{B}=\hat{\tilde{B}}(0)$ are operators considered at the steady state of the system. In the remainder of the paper, we assume that $\hat{U}(\tau) \equiv \hat{U}(\tau, 0)$. Particularly, the Eq. (9) takes the form of the Eq. (1) after performing the integral transformation. More precisely, by taking the real part of the Laplace transform to the Eq. (9), we obtain an expression in terms of the Green's functions operator in the frequency domain as follows

$$
S(\omega)=\mathfrak{R e} \operatorname{Tr}_{S}[\hat{A} \hat{\widetilde{G}}(i \omega)] .
$$

Notice that the operators $\hat{A}$ and $\hat{B}$ should be defined appropriately for describing the emission spectrum due to the cavity or the quantum dot. Moreover, the wide tilde is used to indicate that the Laplace transform was taken. The superscript "(ss)" should be understood to be the steady state of the reduced density operator of the system. After taking the Laplace transform of the Eq. (9), we obtain an expression for the emission spectrum of the system in terms of the Green's functions operator in the frequency domain as follows

$$
S(\omega)=\frac{1}{\pi n_{c}} \mathfrak{R e} \operatorname{Tr}_{S}[\hat{A} \hat{\tilde{G}}(i \omega)] .
$$

Prior to leaving this section, we mention that this result will be the starting point for calculating the emission spectrum due to the cavity as well as the quantum dot by considering the photon and fermionic operators in a separated way.

\section{Application to the QD-cavity system}

\section{i. Model}

In order to illustrate the potential of the Green's function technique for calculating the emission spectrum in a QD-cavity system, we will consider an open quantum system composed of a quantum dot interacting with a confined mode of the electromagnetic field inside a semiconductor cavity. This quantum system is well described by the JaynesCummings Hamiltonian [24]

$$
\hat{H}_{S}=\omega_{X} \hat{\sigma}^{\dagger} \hat{\sigma}+\left(\omega_{X}-\Delta\right) \hat{a}^{\dagger} \hat{a}+g\left(\hat{\sigma} \hat{a}^{\dagger}+\hat{a} \hat{\sigma}^{\dagger}\right),
$$

where the quantum dot is described as a fermionic system with only two possible states, e.g., $|G\rangle$ and $|X\rangle$ are the ground and excited state. $\hat{\sigma}=|G\rangle\langle X|$ and $\hat{a}\left(\hat{\sigma}^{\dagger}=|X\rangle\langle G|\right.$ and $\left.\hat{a}^{\dagger}\right)$ are the annihilation (creation) operators for the fermionic system and the cavity mode, respectively. The parameter $g$ is the light-matter coupling constant. Moreover, note that we have set $\hbar=1$. We also define the detuning between frequencies of the quantum dot and the cavity mode as $\Delta=\omega_{X}-\omega_{a}$, where $\omega_{X}$ and $\omega_{a}$ are the energies associated to an exciton and the photons inside the cavity, respectively. This Hamiltonian system is far from describing any real physical situation since it is completely integrable [25] and no measurements could be done since the light remains always inside the cavity.

In order to incorporate the effects of the environment on the dynamics of the system, we consider the usual approach to model an open quantum system by considering a whole system-reservoir Hamiltonian which is frequently split in three parts. Namely, $\hat{H}=\hat{H}_{S}+\hat{H}_{S R}+\hat{H}_{R}$, where $\hat{H}_{S}$ defines the Hamiltonian term of the QD-cavity system as it is defined in the Eq. (12). The Hamiltonian terms $\hat{H}_{S R}$ and $\hat{H}_{R}$ corresponding to a bilinear coupling between the system-reservoir and its respective reservoirs $\hat{H}_{R}$ have been discussed in detail by Perea et al. in [26]. The reader can find a detailed discussion of the Markovian master equation in $[27,28]$. In the framework of open quantum systems, different reservoirs have been proposed in order to describe the dissipation, decoherence or decays. Particularly, for QD-cavity systems, a reservoir is considered for describing the 
Papers in Physics, vol. 8, ARt. 080008 (2016) / E. A. Gómez et al.

physical situation where the photons are absorbed in a semiconductor and electron-hole pairs (excitons) can be produced which can be associated to either electrical injection or the capture of excitons optically created at frequencies larger than the typical ones of our system. This process corresponds to the so-called continuous and incoherent pumping of the QD. Also, when the excitons are coupled to the leaky modes of the cavity with energy different than the cavity mode, there is a residual density of states inside the cavity and this process is responsible for the spontaneous emission (radiative recombination) to an independent reservoir of photons. Another physical process is known as the coherent emission and it is due to the direct dissipation of the cavity mode, more precisely, the cavity mode is coupled to the continuum of photonic modes out of the cavity. For obtaining the master equation for the QD-cavity system, it is convenient to consider the interaction picture with respect to $\hat{H}_{S}+\hat{H}_{S R}$ and assume the validity of the Born-Markov approximation. After tracing out the degrees of freedom of all the reservoirs, one arrives to the Lindblad master equation for the reduced density matrix of the system

$$
\begin{aligned}
\frac{d \hat{\tilde{\rho}}_{S}}{d \tau} & =-i\left[\hat{H}_{S}, \hat{\tilde{\rho}}_{S}\right] \\
& +\frac{\kappa}{2}\left(2 \hat{a} \hat{\tilde{\rho}}_{S} \hat{a}^{\dagger}-\hat{a}^{\dagger} \hat{a} \hat{\tilde{\rho}}_{S}-\hat{\tilde{\rho}}_{S} \hat{a}^{\dagger} \hat{a}\right) \\
& +\frac{\gamma}{2}\left(2 \hat{\sigma} \hat{\tilde{\rho}}_{S} \hat{\sigma}^{\dagger}-\hat{\sigma}^{\dagger} \hat{\sigma} \hat{\tilde{\rho}}_{S}-\hat{\tilde{\rho}}_{S} \hat{\sigma}^{\dagger} \hat{\sigma}\right) \\
& +\frac{P}{2}\left(2 \hat{\sigma}^{\dagger} \hat{\tilde{\rho}}_{S} \hat{\sigma}-\hat{\sigma} \hat{\sigma}^{\dagger} \hat{\tilde{\rho}}_{S}-\hat{\tilde{\rho}}_{S} \hat{\sigma} \hat{\sigma}^{\dagger}\right) .
\end{aligned}
$$

The parameter $\gamma$ is the decay rate due to the spontaneous emission, $\kappa$ is the decay rate of the cavity photons across the cavity mirrors, and $P$ is the rate at which the excitons are being pumped. Figure 1 shows a scheme of the simplified model of the QDcavity system showing the processes of continuous pumping $P$ and cavity loses $\kappa$. The physical process begins when the light from the pumping laser enters into the cavity and excites one of the quantum dots in the QD layer. Thus, light from this source couples to the cavity and a fraction of photons escapes through the partly transparent mirror from the cavity and goes to the spectrometer for measurements of the emission spectrum.

A general approach for solving the dynamics of the system consists in writing the corresponding Bloch equations for the reduced density matrix of the system in the bared basis. It is an extended Hilbert space formed by taking the tensor product of the state vectors for each of the system components, $\{|G\rangle,|X\rangle\} \otimes\{|n\rangle\}_{n=0}^{\infty}$. In this basis, the reduced density matrix $\hat{\rho}_{S}$ can be written in terms of its matrix elements as $\tilde{\rho}_{S \alpha n, \beta m} \equiv\left\langle\alpha n\left|\hat{\tilde{\rho}}_{S}(\tau)\right| \beta m\right\rangle$. Hence, the Eq. (13) explicitly reads

$$
\begin{aligned}
\frac{d \tilde{\rho}_{S \alpha n, \beta m}}{d \tau} & =i\left[\left(\omega_{X}-\Delta\right)(m-n) \tilde{\rho}_{S \alpha n, \beta m}\right. \\
& \left.+\omega_{X}\left(\delta_{\beta X} \tilde{\rho}_{S \alpha n, X m}-\delta_{\alpha X} \tilde{\rho}_{S X n, \beta m}\right)\right] \\
& +i g\left[\left(\sqrt{m+1} \delta_{\beta X} \tilde{\rho}_{S \alpha n, G m+1}\right.\right. \\
& \left.+\sqrt{m} \delta_{\beta G} \tilde{\rho}_{S \alpha n, X m-1}\right) \\
& -\left(\sqrt{n} \delta_{\alpha G} \tilde{\rho}_{S X n-1, \beta m}\right. \\
& \left.\left.+\sqrt{n+1} \delta_{\alpha X} \tilde{\rho}_{S G n+1, \beta m}\right)\right] \\
& +\frac{\kappa}{2}\left(2 \sqrt{(m+1)(n+1)} \tilde{\rho}_{S \alpha n+1, \beta m+1}\right. \\
& \left.-(n+m) \tilde{\rho}_{S \alpha n, \beta m}\right)-\frac{\gamma}{2}\left(\delta_{\alpha X} \tilde{\rho}_{S X n, \beta m}\right. \\
& \left.-2 \delta_{\alpha G} \delta_{\beta G} \tilde{\rho}_{S X n, X m}+\delta_{\beta X} \tilde{\rho}_{S \alpha n, X m}\right) \\
& +\frac{P}{2}\left(2 \delta_{\alpha X} \delta_{\beta X} \tilde{\rho}_{S G n, G m}-\delta_{\alpha G} \tilde{\rho}_{S G n, \beta m}\right. \\
& \left.-\delta_{\beta G} \tilde{\rho}_{S \alpha n, G m}\right) .
\end{aligned}
$$

Note that we use the convention that all indices written in Greek alphabet are used for the fermionic states and take only two possible values $|G\rangle,|X\rangle$. The indices written in Latin alphabet are used for the Fock states which take the possible values $0,1,2,3 \ldots$ Additionally, it is worth mentioning that our proposed method does not require to solve a system of coupled differential equations, instead of it, we solve a reduced set of algebraic equations that speed up the numerical solution.

Prior to leaving this section, we point out that the number of excitations of the system is defined by the operator $\hat{N}=\hat{a}^{\dagger} \hat{a}+\hat{\sigma}^{\dagger} \hat{\sigma}$. The closed system and the number of excitations of the system is conserved, i.e., $\left[\hat{H}_{S}, \hat{N}\right]=0$. It allows us to organize the states of the system through the number of excitations criterion such that the density matrix elements $\tilde{\rho}_{G n, G n}, \tilde{\rho}_{X n-1, X n-1}, \tilde{\rho}_{G n, X n-1}$ and $\tilde{\rho}_{X n-1, G n}$ are related by having the same number of 


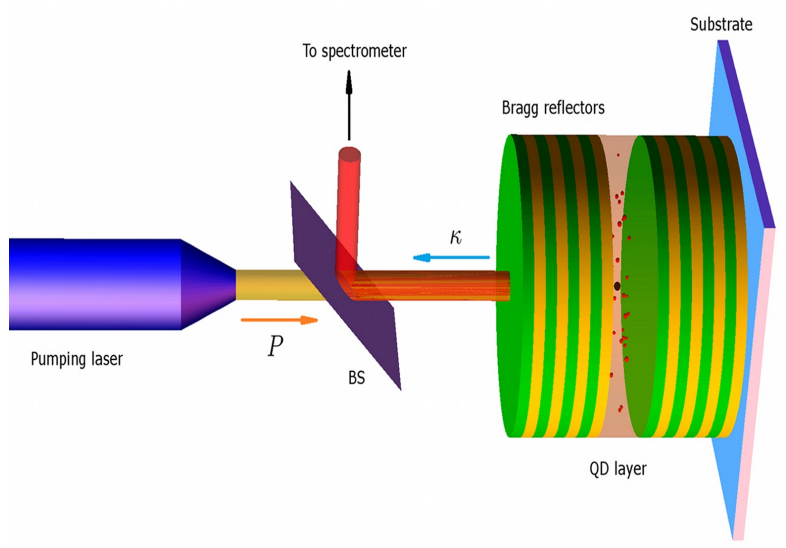

Figure 1: The picture represents a QD-cavity system showing the processes of continuous pumping $P$ and cavity loses $\kappa$.

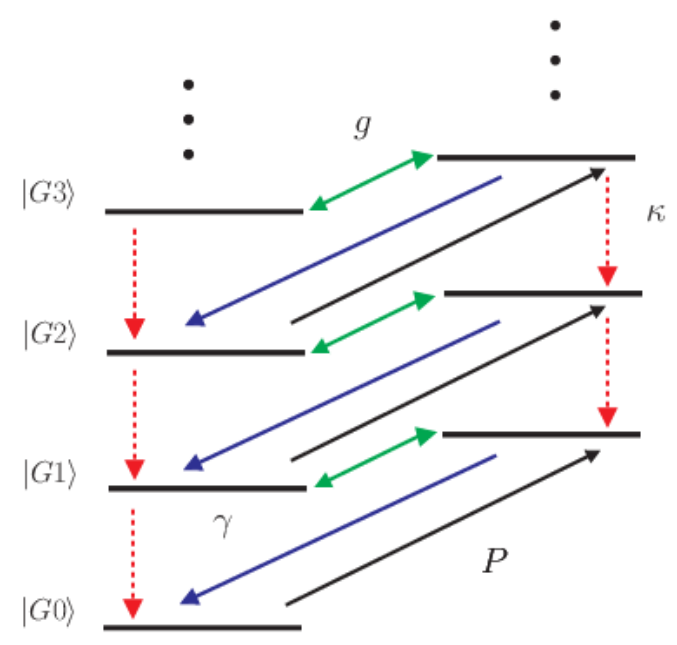

Figure 2: Ladder of bared states for a two-level quantum dot coupled to a single cavity mode. The double headed green arrow depicts the matter coupling constant $g$, dashed red lines the emission of the cavity mode $\kappa$, solid black lines the exciton pumping rate $P$ and solid blue lines the spontaneous emission rate $\gamma$.

quanta; sub-spaces of a fixed number of excitation evolve independently from each other. The Fig. 2 shows a schematic representation of the action of the dissipative processes involved in the dynamics of the system according to the excitation number $\left(N_{\text {exc }}\right)$.

\section{ii. Emission spectrum of the cavity based on the GFT}

In order to compute the emission spectrum of the cavity, we will consider the two-time correlation function accordingly with the Eq. (9) for the field operator as follows

$$
\lim _{t \rightarrow \infty}\left\langle\hat{\tilde{a}}^{\dagger}(t+\tau) \hat{\tilde{a}}(t)\right\rangle=\operatorname{Tr}_{S}\left[\hat{a}^{\dagger} \hat{\tilde{G}}(\tau)\right] .
$$

Where we have considered that the field operator is given by $\hat{\tilde{a}}(0)=\hat{a}$ at the steady state. After performing the partial trace over the degrees of freedom of the system, we have that

$$
\begin{aligned}
\operatorname{Tr}_{S}\left[\hat{a}^{\dagger} \hat{\tilde{G}}(\tau)\right] & =\sum_{\alpha, \beta, \gamma, l, m, n} \sqrt{(l+1)(m+1)} \\
& \times \operatorname{Tr}_{R}\left[U_{\alpha l, \beta m}(\tau)\right. \\
& \left.\times\left\langle\beta m+1\left|\hat{\rho}_{S \otimes R}^{(s)}\right| \gamma n\right\rangle U_{\gamma n, \alpha l+1}^{\dagger}(\tau)\right],
\end{aligned}
$$

where the matrix elements for the time evolution operator are given by $U_{\alpha l, \beta m}(\tau)=\langle\alpha l|\hat{U}(\tau)| \beta m\rangle$ and $U_{\gamma n, \alpha l+1}^{\dagger}(\tau)=\left\langle\gamma n\left|\hat{U}^{\dagger}(\tau)\right| \alpha l+1\right\rangle$. In what follows, we assume the validity of the Markovian approximation, it means that the correlations between the system and the reservoir must be unimportant even at the steady state. Thus, the density operator system-reservoir can be written as $\hat{\rho}_{S \otimes R}^{(s s)}=\hat{\rho}_{S}^{(s s)} \otimes \hat{\rho}_{R}^{(s s)}$ which implies that

$$
\left\langle\beta m+1\left|\hat{\rho}_{S \otimes R}^{(s s)}\right| \gamma n\right\rangle=\hat{\rho}_{R}^{(s s)}\left\langle\beta m+1\left|\hat{\rho}_{S}^{(s s)}\right| \gamma n\right\rangle .
$$

Replacing the previous expression in Eq. (16), it is straightforward to show that the two-time correlation function reads

$$
\operatorname{Tr}_{S}\left[\hat{a}^{\dagger} \hat{\tilde{G}}(\tau)\right]=\sum_{\alpha l} \sqrt{l+1}\langle\alpha l|\hat{\tilde{G}}(\tau)| \alpha l+1\rangle,
$$

where the Green's functions operator $\hat{\tilde{G}}(\tau)$ is given by

$$
\begin{aligned}
\hat{\tilde{G}}(\tau) & =\operatorname{Tr}_{R}\left[\hat{U}(\tau) \hat{\rho}_{R}^{(s s)} \sum_{\beta \gamma m n}(\sqrt{m+1}|\beta m\rangle\langle\gamma n|\right. \\
& \left.\left.\times\left\langle\beta m+1\left|\hat{\rho}_{S}^{(s s)}\right| \gamma n\right\rangle\right) \hat{U}^{\dagger}(\tau)\right]
\end{aligned}
$$


As we pointed out in section II, this operator must obey the same master equation as the reduced density operator of the system. In fact, the terms that only contribute in the Eq. (18) are given by the matrix elements $\tilde{G}_{\beta m, \gamma n}(\tau) \equiv\langle\beta m|\hat{\tilde{G}}(\tau)| \gamma n\rangle$ of the Green's functions operator. This is due to the fact that the projection operator $|\beta m\rangle\langle\gamma n|$ enters into $\hat{\tilde{G}}(\tau)$ in the same way as into the reduced density operator of the system.

In order to identify these matrix elements, it should be considered that for the QD-cavity system, the dynamics of all coherences asymptotically vanish and there only remains the reduced density matrix elements which are ruled by the number of excitations criterion, i.e., $\rho_{G n, G n}, \rho_{X n-1, X n-1}$, $\rho_{G n, X n-1}, \rho_{X n-1, G n}$. Thus, the Eq. (17) can be written as follows

$$
\begin{aligned}
\left\langle\beta m+1\left|\hat{\rho}_{S \otimes R}^{(s s)}\right| \gamma n\right\rangle & =\hat{\rho}_{R}^{(s s)}\left(\delta_{\beta G} \delta_{\gamma G} \delta_{m+1, n}\right. \\
& +\delta_{\beta X} \delta_{\gamma X} \delta_{m, n-1} \\
& +\delta_{\beta G} \delta_{\gamma X} \delta_{m, n} \\
& \left.+\delta_{\beta X} \delta_{\gamma G} \delta_{m+1, n-1}\right) \\
& \times \rho_{S \beta m+1, \gamma n}^{(s s)}
\end{aligned}
$$

By replacing the Eq. (20) into Eq. (19), we find that the Green's functions operator explicitly reads

$$
\begin{aligned}
\hat{\tilde{G}}(\tau) & =\operatorname{Tr}_{R}\left[\hat{U}(\tau) \hat{\rho}_{R}^{(s s)} \sum_{m} \sqrt{m+1}\right. \\
& \times\left(|G m\rangle\langle G m+1| \rho_{S G m+1, G m+1}^{(s s)}\right. \\
& +|X m\rangle\langle X m+1| \rho_{S X m+1, X m+1}^{(s s)} \\
& +|G m\rangle\langle X m| \rho_{S G m+1, X m}^{(s s)} \\
& +|X m\rangle\langle G m+2| \\
& \left.\left.\times \rho_{S X m+1, G m+2}^{(s s)}\right) \hat{U}^{\dagger}(\tau)\right]
\end{aligned}
$$

Note that from this expression, it is easy to identify the nonzero matrix elements of the Green's functions operator that contribute to the emission spectrum. Finally, after performing the Laplace transform to the Eq. (18), we have that the emission spectrum of the cavity is given by

$$
\begin{aligned}
S(\omega) & =\mathfrak{R e} \sum_{l} \sqrt{l+1}\left(\widetilde{G}_{G l, G l+1}(i \omega)\right. \\
& +\widetilde{G}_{X l, X l+1}(i \omega)+\widetilde{G}_{G l, X l}(i \omega) \\
& \left.+\widetilde{G}_{X l, G l+2}(i \omega)\right) .
\end{aligned}
$$

It is worth mentioning that the initial conditions may be obtained by evaluating the Green's function operator at $\tau=0$. Moreover, by using the fact that the time evolution operators become the identity and $\operatorname{Tr}_{R}\left[\hat{\rho}_{R}^{(s s)}\right]=1$. We obtain a set of initial conditions given by

$$
\begin{aligned}
\tilde{G}_{G l, G l+1}(0) & =\sqrt{l+1} \rho_{S G l+1, G l+1}^{(s s)}, \\
\tilde{G}_{X l, X l+1}(0) & =\sqrt{l+1} \rho_{S X l+1, X l+1}^{(s s)}, \\
\tilde{G}_{G l, X l}(0) & =\sqrt{l+1} \rho_{S G l+1, X l}^{(s s)}, \\
\tilde{G}_{X l, G l+2}(0) & =\sqrt{l+1} \rho_{S X l+1, G l+2}^{(s s)} .
\end{aligned}
$$

Note that this set of initial conditions corresponds to the steady state of the reduced density matrix of the system. A general algorithm based on the GFT for computing the emission spectrum is presented in the appendix. We mention that this approach can be adapted easily for calculating the emission spectrum due to the cavity as well as the quantum dot.

\section{iii. Emission spectrum of the quantum dot based on the GFT}

In order to compute the emission spectrum of the quantum dot, we will consider the two-time correlation function given by Eq. (9), but for the case of the matter operator

$$
\lim _{t \rightarrow \infty}\left\langle\hat{\tilde{\sigma}}^{\dagger}(t+\tau) \hat{\tilde{\sigma}}(t)\right\rangle=\operatorname{Tr}_{S}\left[\hat{\sigma}^{\dagger} \hat{\tilde{G}}(\tau)\right]
$$

where we have considered that the matter operator is given by $\hat{\tilde{\sigma}}(0)=\hat{\sigma}$ at the steady state. It is straightforward to show, after performing the partial trace over the degrees of freedom of the system, that the two-time correlation function reads

$$
\operatorname{Tr}_{S}\left[\hat{\sigma}^{\dagger} \hat{\tilde{G}}(\tau)\right]=\sum_{\alpha l} \delta_{\alpha X}\langle G l|\hat{\tilde{G}}(\tau)| \alpha l\rangle,
$$


where the Green's functions operator $\hat{\tilde{G}}(\tau)$ is given by

$$
\begin{aligned}
\hat{\tilde{G}}(\tau) & =\operatorname{Tr}_{R}\left[\hat { U } ( \tau ) \sum _ { \beta \gamma m n } \left(\delta_{\beta X}|G m\rangle\langle\gamma m|\right.\right. \\
& \left.\left.\times\left\langle\beta m\left|\hat{\rho}_{S \otimes R}^{(s s)}\right| \gamma n\right\rangle\right) \hat{U}^{\dagger}(\tau)\right] .
\end{aligned}
$$

Assuming again the validity of the Markovian approximation and taking into account the number of excitations criterion, we have that the density operator system-reservoir can be written as

$$
\begin{aligned}
\left\langle\beta m\left|\hat{\rho}_{S \otimes R}^{(s s)}\right| \gamma n\right\rangle & =\hat{\rho}_{R}^{(s s)}\left(\delta_{\beta G} \delta_{\gamma G} \delta_{m, n}\right. \\
& +\delta_{\beta X} \delta_{\gamma X} \delta_{m, n}+\delta_{\beta G} \delta_{\gamma X} \delta_{m, n+1} \\
& \left.+\delta_{\beta X} \delta_{\gamma G} \delta_{m, n-1}\right) \rho_{S \beta m, \gamma n}^{(s s)} .(27)
\end{aligned}
$$

By inserting the Eq. (27) into Eq. (26), we find that the Green's functions operator explicitly reads

$$
\begin{aligned}
\hat{\tilde{G}}(\tau) & =\operatorname{Tr}_{R}\left[\hat{U}(\tau) \hat{\rho}_{R}^{(s s)}\right. \\
& \times \sum_{m}\left(|G m\rangle\langle X m| r h o_{S X m, X m}^{(s s)}\right. \\
& \left.+|G m\rangle\langle G m+1| \rho_{S X m, G m+1}^{(s s)}\right) \\
& \left.\times \hat{U}^{\dagger}(\tau)\right] .
\end{aligned}
$$

Analogously as in section ii, we identify the nonzero matrix elements of the Green's functions operator that contribute to the emission spectrum. After performing the Laplace transform to Eq. (25), the emission spectrum of the quantum dot is given by

$$
S(\omega)=\mathfrak{R e} \sum_{l}\left(\widetilde{G}_{G l, X l}(i \omega)+\widetilde{G}_{G l, G l+1}(i \omega)\right) .
$$

Taking into account that the initial conditions are obtained by evaluating the Green's function operator at $\tau=0$, we have the time evolution operators become the identity and $\operatorname{Tr}_{R}\left[\hat{\rho}_{R}^{(s s)}\right]=1$, thus, we obtain a set of initial conditions given by

$$
\begin{aligned}
\tilde{G}_{G l, X l}(0) & =\rho_{S X l, X l}^{(s s)}, \\
\tilde{G}_{G l, G l+1}(0) & =\rho_{S X l, G l+1}^{(s s)}, \\
\tilde{G}_{X l, X l+1}(0) & =0, \\
\tilde{G}_{G l+2, X l}(0) & =0 .
\end{aligned}
$$

at the steady-state.

\section{iv. Emission spectrum of the cavity based on the QRT}

In what follows, we apply the QRT approach for the model of QD-cavity system described in section III. In order to compute the emission spectrum of the cavity, the knowledge of the two-time correlation function for the field operator is required, it is $\left\langle\hat{a}^{\dagger}(\tau) \hat{a}(0)\right\rangle$ in concordance with the Eq. (1). Moreover, by following the approach presented in Ref. [26], it is straightforward to show that the twotime correlation function is given by

$$
\begin{aligned}
\left\langle\hat{a}^{\dagger}(\tau) \hat{a}(0)\right\rangle & =\sum_{n} \sqrt{n+1}\left(\left\langle\hat{a}_{G n}^{\dagger}(\tau) \hat{a}(0)\right\rangle\right. \\
& \left.+\left\langle\hat{a}_{X n}^{\dagger}(\tau) \hat{a}(0)\right\rangle\right)
\end{aligned}
$$

where the following definitions have been used

$$
\begin{aligned}
\hat{a}_{G n}^{\dagger} & =|G n+1\rangle\langle G n|, \\
\hat{a}_{X n}^{\dagger} & =|X n+1\rangle\langle X n|, \\
\hat{\sigma}_{n}^{\dagger} & =|X n\rangle\langle G n|, \\
\hat{\zeta}_{n} & =|G n+1\rangle\langle X n-1| .
\end{aligned}
$$

It is worth mentioning that the last two operators should be added in order to close the dynamics of the system accordingly to the QRT as pointed out in section II. More precisely, we are interested in solving the dynamical equations associated to the expectation values $\left\langle\hat{a}_{G n}^{\dagger}(\tau) \hat{a}(0)\right\rangle$ and $\left\langle\hat{a}_{X n}^{\dagger}(\tau) \hat{a}(0)\right\rangle$ as a function of $\tau$. Therefore, we need to solve a set of coupled differential equations given by

$$
\begin{aligned}
\frac{d}{d \tau}\left\langle\hat{a}_{G n}^{\dagger}(\tau) \hat{a}(0)\right\rangle & =\sum_{j} L_{i j}\left\langle\hat{a}_{G n}^{\dagger}(\tau) \hat{a}(0)\right\rangle, \\
\frac{d}{d \tau}\left\langle\hat{a}_{X n}^{\dagger}(\tau) \hat{a}(0)\right\rangle & =\sum_{j} L_{i j}\left\langle\hat{a}_{X n}^{\dagger}(\tau) \hat{a}(0)\right\rangle, \\
\frac{d}{d \tau}\left\langle\hat{\sigma}_{n}^{\dagger}(\tau) \hat{a}(0)\right\rangle & =\sum_{j} L_{i j}\left\langle\hat{\sigma}_{n}^{\dagger}(\tau) \hat{a}(0)\right\rangle, \\
\frac{d}{d \tau}\left\langle\hat{\zeta}_{n}(\tau) \hat{a}(0)\right\rangle & =\sum_{j} L_{i j}\left\langle\hat{\zeta}_{n}(\tau) \hat{a}(0)\right\rangle .
\end{aligned}
$$

In order to find explicitly the set of dynamical equations and its corresponding initial conditions, 
we obtain first the set of differential equations for the single-time expectation values for the operators given by Eq. (32), it is explicitly

$$
\begin{aligned}
\frac{d}{d \tau}\left\langle\hat{a}_{G n}^{\dagger}(\tau)\right\rangle & =\left(-P-i \Delta-n \kappa-\frac{\kappa}{2}+i \omega_{X}\right) \\
& \times\left\langle\hat{a}_{G n}^{\dagger}(\tau)\right\rangle+\kappa \sqrt{(n+1)(n+2)} \\
& \times\left\langle\hat{a}_{G n+1}^{\dagger}(\tau)\right\rangle+\gamma\left\langle\hat{a}_{X n}^{\dagger}(\tau)\right\rangle \\
& -i g \sqrt{n}\left\langle\hat{\zeta}_{n}(\tau)\right\rangle+i g \sqrt{n+1} \\
& \times\left\langle\hat{\sigma}_{n}^{\dagger}(\tau)\right\rangle \\
\frac{d}{d \tau}\left\langle\hat{\sigma}_{n}^{\dagger}(\tau)\right\rangle & =i g \sqrt{n+1}\left\langle\hat{a}_{G n}^{\dagger}(\tau)\right\rangle \\
& -i g \sqrt{n}\left\langle\hat{a}_{X n-1}^{\dagger}(\tau)\right\rangle \\
& +\frac{1}{2}\left(-P-\gamma-2 n \kappa+2 i \omega_{X}\right) \\
& \times\left\langle\hat{\sigma}_{n}^{\dagger}(\tau)\right\rangle \\
& +(n+1) \kappa\left\langle\hat{\sigma}_{n+1}^{\dagger}(\tau)\right\rangle, \\
& =P\left\langle\hat{a}_{G n-1}^{\dagger}(\tau)\right\rangle+\kappa \sqrt{n(n+1)} \\
& \times\left\langle\hat{a}_{X n}^{\dagger}(\tau)\right\rangle \\
& +\left(-\gamma-i \Delta-n \kappa+\frac{\kappa}{2}+i \omega_{X}\right) \\
& \times\left\langle\hat{a}_{X n-1}^{\dagger}(\tau)\right\rangle \\
& -i g \sqrt{n}\left\langle\hat{\sigma}_{n}^{\dagger}(\tau)\right\rangle, \\
& =-i g \sqrt{n}\left\langle\hat{a}_{G n}^{\dagger}(\tau)\right\rangle+i g \sqrt{n+1} \\
& \times\left\langle\hat{a}_{X n-1}^{\dagger}(\tau)\right\rangle \\
& +\left(-\frac{P}{2}-\frac{\gamma}{2}-2 i \Delta-n \kappa+i \omega_{X}\right) \\
& \times\left\langle\hat{\zeta}_{n}(\tau)\right\rangle \\
& +\sqrt{n(n+2)} \kappa\left\langle\hat{\zeta}_{n+1}(\tau)\right\rangle . \\
\frac{d}{d \tau}\left\langle\hat{\zeta}_{n}(\tau)\right\rangle & (34) \\
&
\end{aligned}
$$

The QRT approach implies that the following two-time correlation functions $\left\langle\hat{a}_{G n}^{\dagger}(\tau) \hat{a}(0)\right\rangle$, $\left\langle\hat{a}_{X n}^{\dagger}(\tau) \hat{a}(0)\right\rangle,\left\langle\hat{\sigma}_{n}^{\dagger}(\tau) \hat{a}(0)\right\rangle$ and $\left\langle\hat{\zeta}_{n}(\tau) \hat{a}(0)\right\rangle$ satisfy the same dynamical equations given by Eq. (34), subject to the initial conditions

$$
\begin{aligned}
\left\langle\hat{a}_{G n}^{\dagger}(0) \hat{a}(0)\right\rangle & =\sqrt{n+1} \rho_{G n+1, G n+1}(0) \\
\left\langle\hat{a}_{X n}^{\dagger}(0) \hat{a}(0)\right\rangle & =\sqrt{n+1} \rho_{X n+1, X n+1}(0), \\
\left\langle\hat{\sigma}_{n}^{\dagger}(0) \hat{a}(0)\right\rangle & =\sqrt{n+1} \rho_{G n+1, X n}(0) \\
\left\langle\hat{\zeta}_{n}(0) \hat{a}(0)\right\rangle & =\sqrt{n} \rho_{X n, G n+1}(0)
\end{aligned}
$$

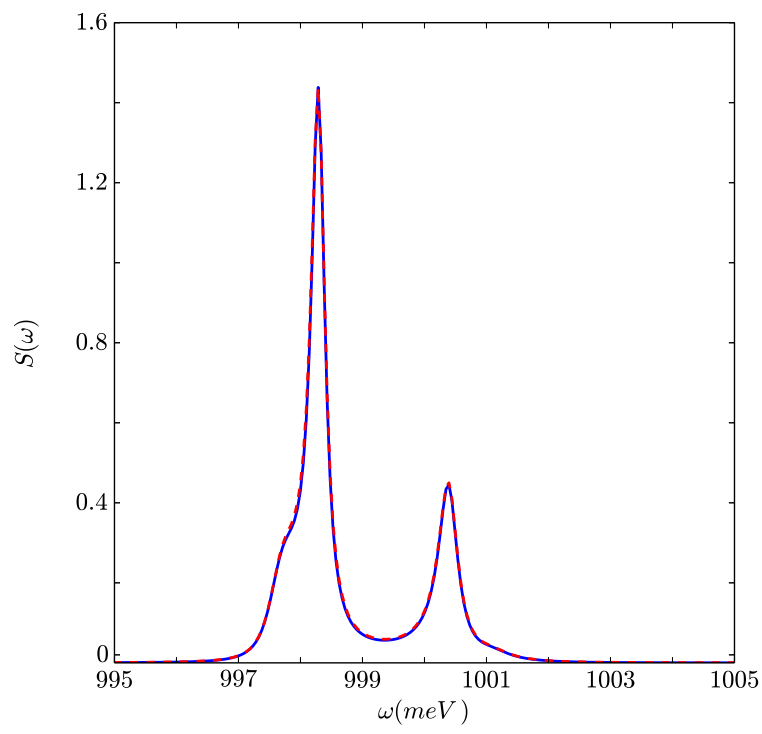

Figure 3: Emission spectrum of the cavity based on the GFT as a solid blue line and the corresponding numerical calculation based on the QRT as a dashed red line. The parameters values are $g=1$ $\mathrm{meV}, \gamma=0.005 \mathrm{meV}, \kappa=0.2 \mathrm{meV}, P=0.3 \mathrm{meV}$, $\Delta=2 \mathrm{meV}, \omega_{a}=1000 \mathrm{meV}$.

More precisely, it is done explicitly by performing the following replacements: $\left\langle\hat{a}_{G n}^{\dagger}(\tau)\right\rangle \rightarrow$ $\left\langle\hat{a}_{G n}^{\dagger}(\tau) \hat{a}(0)\right\rangle, \quad\left\langle\hat{a}_{X n}^{\dagger}(\tau)\right\rangle \rightarrow\left\langle\hat{a}_{X n}^{\dagger}(\tau) \hat{a}(0)\right\rangle$, $\left\langle\hat{\sigma}_{n}^{\dagger}(\tau)\right\rangle \rightarrow\left\langle\hat{\sigma}_{n}^{\dagger}(\tau) \hat{a}(0)\right\rangle$ and $\left\langle\hat{\zeta}_{n}(\tau)\right\rangle \rightarrow\left\langle\hat{\zeta}_{n}(\tau) \hat{a}(0)\right\rangle$. The parameters of the system $\omega_{X}, \Delta, g, \kappa, \gamma$ determine the dynamics of the two-time correlation function, as well as setting the initial conditions that will be propagated according to the dynamical equations given by Eq. (34). Since we are interested in the light that the quantum system emits, we have considered the steady state of the system as the initial state into equation Eq. (35).

\section{Results and discussion}

In this section, we compare the numerical calculations based on the GFT and the QRT approach for the emission spectrum of the cavity as well as the quantum dot. In particular, the QD-cavity system can display two different dynamical regimes by changing the parameters of the system and two regimes can be achieved when the loss and 
Papers in Physics, vol. 8, ART. 080008 (2016) / E. A. Gómez et al.

pump rates are modified. In fact, the relation $g>|\kappa-\gamma| / 4$ holds for the strong coupling regime and the relation $g<|\kappa-\gamma| / 4$ remains valid for the weak coupling regime. Figure 3 shows the numerical results for the emission spectrum associated to the cavity in the strong coupling regime, where the emission spectrum of the cavity based on the GFT is shown as a solid blue line and the emission spectrum based on the QRT approach as a dashed red line. The parameters of the system are $g=1 \mathrm{meV}$, $\gamma=0.005 \mathrm{meV}, \kappa=0.2 \mathrm{meV}, P=0.3 \mathrm{meV}, \Delta=2$ $\mathrm{meV}$ and $\omega_{a}=1000 \mathrm{meV}$. Particularly, for this set of parameters values, we can identify two different peaks which are associated to the energy of the cavity and the quantum dot, they are $\omega_{a} \approx 998.3 \mathrm{meV}$ and $\omega_{X} \approx 1000.3 \mathrm{meV}$. We have considered the relative error as a quantitative measure of the discrepancy between the GFT and the QRT approaches. More precisely, by monitoring the numerical computations of the emission spectrum, we have estimated that the maximum relative error is on the order of $10^{-3}$ in all numerical calculations that we have performed. Similarly, the emission spectrum of the cavity based on the GFT (solid blue line) and the QRT approach (dashed red line) for the strong coupling regime are shown in Fig. 4. Here, the parameters values of the system are given by $g=1 \mathrm{meV}, \gamma=0.005 \mathrm{meV}, \kappa=2 \mathrm{meV}, P=0.005$ $\mathrm{meV}, \Delta=0.0 \mathrm{meV}$ and $\omega_{a}=1000 \mathrm{meV}$. Note that we have considered the resonant case, more precisely, the same energy values for the cavity and the quantum dot. Here, the emission spectrums do not match but repel each other, resulting in a structure of two separate peaks for a distance of approximately two times the coupling constant, i.e., $2 g \approx 2$ $\mathrm{meV}$. It is worth mentioning that this quantum effect is well-known as Rabi splitting in QD-cavity systems. The emission spectrum of the quantum dot in the weak coupling regime is shown in Fig. 5. The numerical result for the emission spectrum of the quantum dot based on the GFT is shown as a solid blue line and the corresponding numerical result for QRT approach is shown as a dashed red line. We set the weak coupling regime by considering high values of the decay and pump rates $\kappa=5$ $\mathrm{meV}$ and $P=1 \mathrm{meV}$, respectively. The rest of the parameters values are $g=1 \mathrm{meV}, \gamma=0.1 \mathrm{meV}$, $\Delta=5 \mathrm{meV}$ and $\omega_{a}=1000 \mathrm{meV}$. We conclude that the method based on the GFT is in perfect agreement with the QRT approach and reproduces

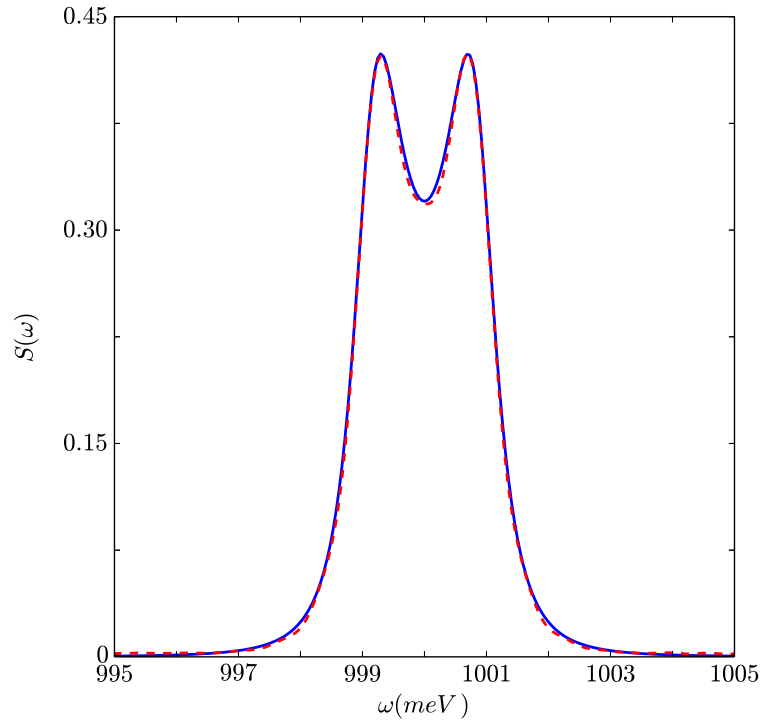

Figure 4: Emission spectrum of the cavity based on the GFT as a solid blue line and the corresponding numerical calculation based on the QRT approach as a dashed red line. The parameters values are $g=1 \mathrm{meV}, \gamma=0.005 \mathrm{meV}, \kappa=2 \mathrm{meV}, P=0.005$ $\mathrm{meV}, \Delta=0 \mathrm{meV}, \omega_{a}=1000 \mathrm{meV}$.

very well the emission spectrum of the QD-cavity system.

For comparison purposes with our GFT approach, we have also implemented the numerical method based on the QRT for the QD-cavity system (see details in section iv). In the conducted simulations, we have considered the same truncation level in the bare-state basis, e.g., $N_{e x c}=10$. Moreover, we have solved numerically the dynamical equations of the system given by the Eq. (34) until time $t_{\max }=2^{17}$ ps for obtaining an acceptable resolution in frequency domain, it is $\Delta \omega \approx 0.048 \mathrm{meV}$. In order to test the performance of the GFT approach in terms of efficiency, we have compared the computational time involved on the numerical calculation of the emission spectrum of the cavity at four different excitation numbers $N_{\text {exc }}$. Table 1 shows in first column the excitation number. Second and third columns show the elapsed time (CPU time) in seconds during the simulations for the GFT and the QRT approach, respectively. It is worth mentioning that we have considered, for this comparison, exactly the same 
Table 1: Comparison of computational time (CPU time) between the Green's Functions Technique (GFT) and the Quantum Regression Theorem (QRT) approach for the numerical calculation of the emission spectrum of the cavity. The computational times were made using a commercial Intel(R) Core(TM) $i 7-4770$ processor of $3.4 \mathrm{GHz} \times 8$, and 12 GB RAM.

\begin{tabular}{ccc}
\hline \hline $\begin{array}{c}\text { Excitation } \\
\text { number }\left(N_{\text {exc }}\right)\end{array}$ & $\begin{array}{c}\text { CPU time(s) } \\
\text { for the GFT }\end{array}$ & $\begin{array}{c}\text { CPU time(s) } \\
\text { for the QRT }\end{array}$ \\
\hline 5 & 0.4 & 92.5 \\
10 & 2.0 & 273.4 \\
20 & 14.0 & 390.2 \\
40 & 100.2 & 673.8 \\
\hline \hline
\end{tabular}

resolution in the frequency domain and the numerical calculations were carried out with the same parameters values as in Fig. 4 for both methods. It is straightforward to observe that the QRT approach is time-consuming compared with the GFT approach when the excitation number is increased. From the computational point of view, it is due to the fact that the QRT approach requires solving a large number of coupled differential equations in contrast to the GFT approach which requires a relatively small system of algebraic equations.

\section{Conclusions}

We have presented the GFT as an alternative methodology to the QRT approach for calculating the two-time correlation functions in open quantum systems. We have applied the GFT and the QRT approach for calculating the emission spectrum in a QD-cavity system. In particular, the performance of the GFT in terms of accuracy and efficiency by comparison of the emission spectrum of the cavity and the quantum dot is demonstrated, as well as by comparison of the computational times involved during the numerical simulations. In fact, we have shown that the GFT offers a computational advantage, namely, the speeding up numerical calculations. We conclude that the GFT allows to overcome the inherent theoretical difficulties presented

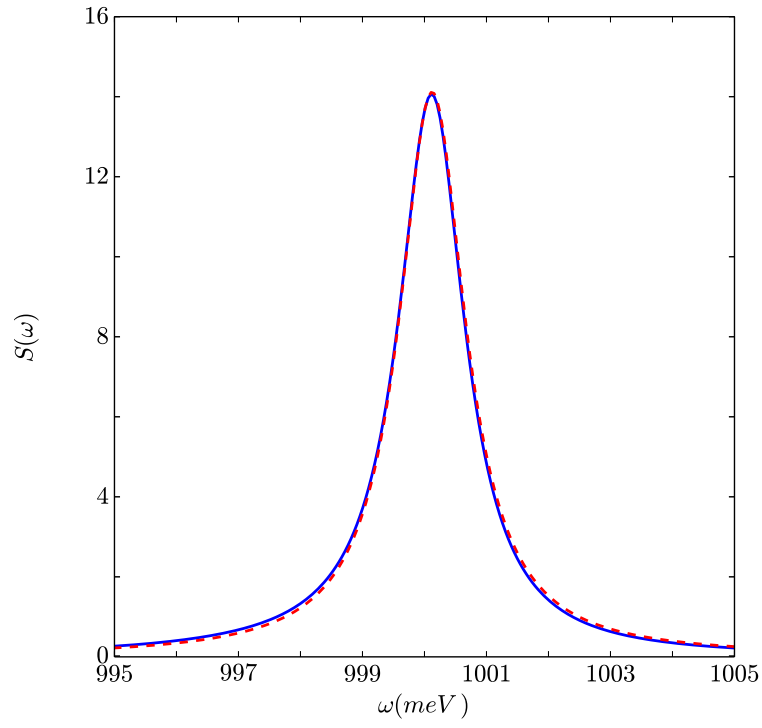

Figure 5: Emission spectrum of the quantum dot based on the GFT as a solid blue line and the corresponding numerical calculation based on the QRT approach as a dashed red line. The parameters values are $\kappa=5 \mathrm{meV}, P=1 \mathrm{meV}, g=1 \mathrm{meV}, \gamma=0.1$ $\mathrm{meV}, \Delta=5 \mathrm{meV}$ and $\omega_{a}=1000 \mathrm{meV}$

in the QRT approach, i.e., to find a closure condition on the set of operators involved in the dynamical equations. We mention that our methodology based on the GFT can be extended for calculating the emission spectrum in significant situations where the quantum dots are in biexcitonic regime or when the quantum dots are coupled to photonic cavities.

Acknowledgements - EAG acknowledges the finacial support from Vicerrectoría de Investigaciones at Universidad del Quindío through research grant No. 752. HVP acknowledges the financial support from Colciencias, within the project with code 11017249692, and HERMES code 31361.

\section{Appendix}

The emission spectrum in a QD-cavity system can easily be computed by taking into account that the dynamics of the operators $\hat{G}(\tau)$ and $\hat{\rho}_{S}(\tau)$ are gov- 
Papers in Physics, vol. 8, Art. 080008 (2016) / E. A. Gómez et al.

erned by the same Linblad master equation, i.e., $d \hat{G}(\tau) / d \tau=\mathcal{L} \hat{G}(\tau)$ with $\mathcal{L}$ the Liouvillian superoperator discussed in section II. Moreover, it has effectively a larger tensor rank than the reduced density operator of the system. Thus, we can write the dynamical equations for the Green's functions operator in a component form

$$
\frac{d G_{\tilde{\alpha}}(\tau)}{d \tau}=\sum_{\tilde{\beta}} \mathcal{L}_{\tilde{\alpha} \tilde{\beta}} G_{\tilde{\beta}}(\tau),
$$

together with the initial condition $G_{\tilde{\beta}}(0)$. Here, the symbol $\tilde{\alpha}$ corresponds to a composite index for labeling the states of the reduced density operator of the system, e.g., for indexing both matter and photon states in the QD-cavity system, see section III. for details. It is worth mentioning that $G_{\tilde{\beta}}$ and $\mathcal{L}_{\tilde{\alpha} \tilde{\beta}}$ act as a column vector and a matrix in this notation. In order to obtain the solution to the Eq. (36) in frequency domain, we perform a Laplace Transform and it explicitly takes the form $-\tilde{G}_{\tilde{\alpha}}(0)=\sum_{\tilde{\beta}}\left(\mathcal{L}_{\tilde{\alpha} \tilde{\beta}}-i \omega \delta_{\tilde{\alpha} \tilde{\beta}}\right) \tilde{G}_{\tilde{\beta}}(i \omega)$. It is straightforward to obtain the solution by performing the matrix inversion to $\mathcal{M}_{\tilde{\alpha} \tilde{\beta}}=\left(i \omega \delta_{\tilde{\alpha} \tilde{\beta}}-\mathcal{L}_{\tilde{\alpha} \tilde{\beta}}\right)$ and the emission spectrum is computed easily in terms of the initial conditions given by

$$
\tilde{G}_{\tilde{\beta}}(i \omega)=\sum_{\tilde{\alpha}} \mathcal{M}_{\tilde{\beta} \tilde{\alpha}}^{-1} \tilde{G}_{\tilde{\alpha}}(0) .
$$

The initial conditions are obtained by evaluating the Green's function operator at $\tau=0$.

[1] H Walther et al., Cavity quantum electrodynamics, Rep. Prog. Phys. 69, 395603 (2006).

[2] A Kavokin, J J Baumberg, G Malpuech, F P Laussy, Microcavities, Oxford University Press (2007).

[3] H Altug, D Englund, J Vuckovic, Ultrafast photonic crystal nanocavity laser, Nat. Phys. 2, 484 (2006).

[4] Y Mu, C M Savage, One-atom lasers, Phys. Rev. A 46, 5944 (1992).

[5] R M Stevenson, R J Young, P Atkinson, K Cooper, D A Ritchie, A J Shields, A semiconductor source of triggered entangled photon pairs, Nature 439, 179 (2006).
[6] T M Stace, G J Milburn, C H W Barnes, Entangled two-photon source using biexciton emission of an asymmetric quantum dot in a cavity, Phys. Rev. B 67, 085317 (2003).

[7] N Gisin, G Ribordy, W Tittel, H Zbinden, Quantum cryptography, Rev. Mod. Phys. 74, 145 (2002).

[8] C Monroe, Quantum information processing with atoms and photons, Nature 416, 238 (2002).

[9] Y Todorov, I Sagnes, I Abram, C Minot, Purcell enhancement of spontaneous emission from quantum cascades inside mirror-grating metal cavities at THz frequencies, Phys. Rev. Lett. 99, 223603 (2007).

[10] J Wiersig et al., Direct observation of correlations between individual photon emission events of a microcavity laser, Nature 460, 245 (2009).

[11] G Khitrova et al., Vacuum Rabi splitting in semiconductors, Nat. Phys. 2, 81 (2006).

[12] J P Reithmaier et al., Strong coupling in a single quantum dot-semiconductor microcavity system, Nature 432, 197 (2004).

[13] L Mandel, E Wolf, Optical Coherence and Quantum Optics, Cambridge University Press, (1997).

[14] O Jedrkiewicz, R Loudon, Atomic dynamics in microcavities: absorption spectra by Green function method, J. Opt. B. - Quantum S. O. 2, R47 (2000).

[15] N V Hieu, N B Ha, Time-resolved luminescence of the coupled quantum dotmicrocavity system: general theory, Adv. Nat. Sci.: Nanosci. Nanotechnol. 1, 045001 (2010).

[16] D F Walls, G J Milburn, Quantum Optics, Springer-Verlag, Berlin (1994).

[17] M Lax, Quantum Noise. IV. Quantum Theory of Noise Sources, Phys. Rev. 145, 110 (1966).

[18] S Swain, Master equation derivation of quantum regression theorem, J. Phys. A. - Math. Gen. 14, 2577 (1981). 
Papers in Physics, vol. 8, ART. 080008 (2016) / E. A. Gómez et al.

[19] E del Valle, F P Laussy, C Tejedor, Luminescence spectra of quantum dots in microcavities. II. Fermions, Phys. Rev. B 79, 235326 (2009).

[20] N Quesada, H Vinck-Posada, B A Rodríguez, Density operator of a system pumped with polaritons: a Jaynes-Cummings-like approach, J. Phys. Condens. Matter 23, 025301 (2011).

[21] C A Vera, N Quesada, H Vinck-Posada, B A Rodríguez, Characterization of dynamical regimes and entanglement sudden death in a microcavity quantum dot system, J. Phys. Condens. Matter 21, 395603 (2009).

[22] N Ishida, T Byrnes, F Nori, Y Yamamoto, Photoluminescence of a microcavity quantum dot system in the quantum strong-coupling regimes, Sci. Rep. 3, 1180 (2013).

[23] T Quang, G S Agarwal, J Bergou, M O Scully, $\mathrm{H}$ Walther, K Vogel, W P Schleich, Calcula- tion of the micromaser spectrum. I. Greensfunction approach and approximate analytical techniques, Phys. Rev. A 48, 803 (1993).

[24] E T Jaynes, F W Cummings, Comparison of quantum and semiclassical radiation theories with application to the beam maser, Proc. IEEE 51, 89 (1963).

[25] M O Scully, M S Zubairy, Quantum Optics, Cambridge, Cambridge University Press (1996).

[26] J I Perea, D Porras, C Tejedor, Dynamics of the excitations of a quantum dot in a microcavity, Phys. Rev. B 70, 115304 (2004).

[27] H P Breuer, F Petruccione, The theory of open quantum systems, Oxford, University Press (2002).

[28] A Rivas, S F Huelga, Open Quantum Systems, Springer (2012). 